\title{
Elli Köngäs Maranda (1932-1982)
}

Centre interuniversitaire d'Études sur les Lettres, les Arts et les Traditions (CÉLAT)

Faculté des Lettres, Université Laval

\section{(1981)}

\section{"La tradition des uni- tés et les unités de la tradition”}

Un document produit en version numérique par Jean-Marie Tremblay, bénévole, professeur de sociologie au Cégep de Chicoutimi

Courriel: jean-marie tremblay@uqac.ca

Site web pédagogique : http://www.uqac.ca/jmt-sociologue/

Dans le cadre de la collection: "Les classiques des sciences sociales"

Site web: http://www.uqac.ca/Classiques_des_sciences_sociales/

Une collection développée en collaboration avec la Bibliothèque Paul-Émile-Boulet de l'Université du Québec à Chicoutimi Site web: http://bibliotheque.uqac.ca/ 
Cette édition électronique a été réalisée par Jean-Marie Tremblay, bénévole, professeur de sociologie au Cégep de Chicoutimi à partir de l'article de :

Elli Köngäs Maranda (1932-1982)

Centre interuniversitaire d’Études sur les Lettres, les Arts et les Traditions (CÉLAT), Faculté des Lettres, Université Laval (1978)

“La tradition des unités et les unités de la tradition”.

Un article publié dans la revue Anthropologie et Sociétés, vol. 5 no 2, 1981, pp. 155-176. Numéro intitulé : “La dynamique biosociale”. Québec : Département d'anthropologie, Université Laval.

[Autorisation formelle accordée, le 6 juillet 2005, par M. Pierre Maranda de diffuser ses travaux ainsi que tous ceux de sa défunte épouse, Mme Elli Köngäs Maranda.]

Courriel pmaranda@videotron.ca

Polices de caractères utilisée :

Pour le texte: Times New Roman, 14 points.

Pour les citations : Times New Roman 12 points.

Pour les notes de bas de page : Times New Roman, 12 points.

Édition électronique réalisée avec le traitement de textes Microsoft Word 2004 pour Macintosh.

Mise en page sur papier format : LETTRE (US letter), 8.5'’ $\mathrm{x}$ 11'”)

Édition complétée le 11 juillet 2007 à Chicoutimi, Ville de Saguenay, province de Québec. 


\section{Elli Köngäs Maranda (1932-1982)}

Centre interuniversitaire d'Études sur les Lettres, les Arts et les Traditions (CÉLAT), Faculté des Lettres, Université Laval (1978)

“La tradition des unités et les unités de la tradition”

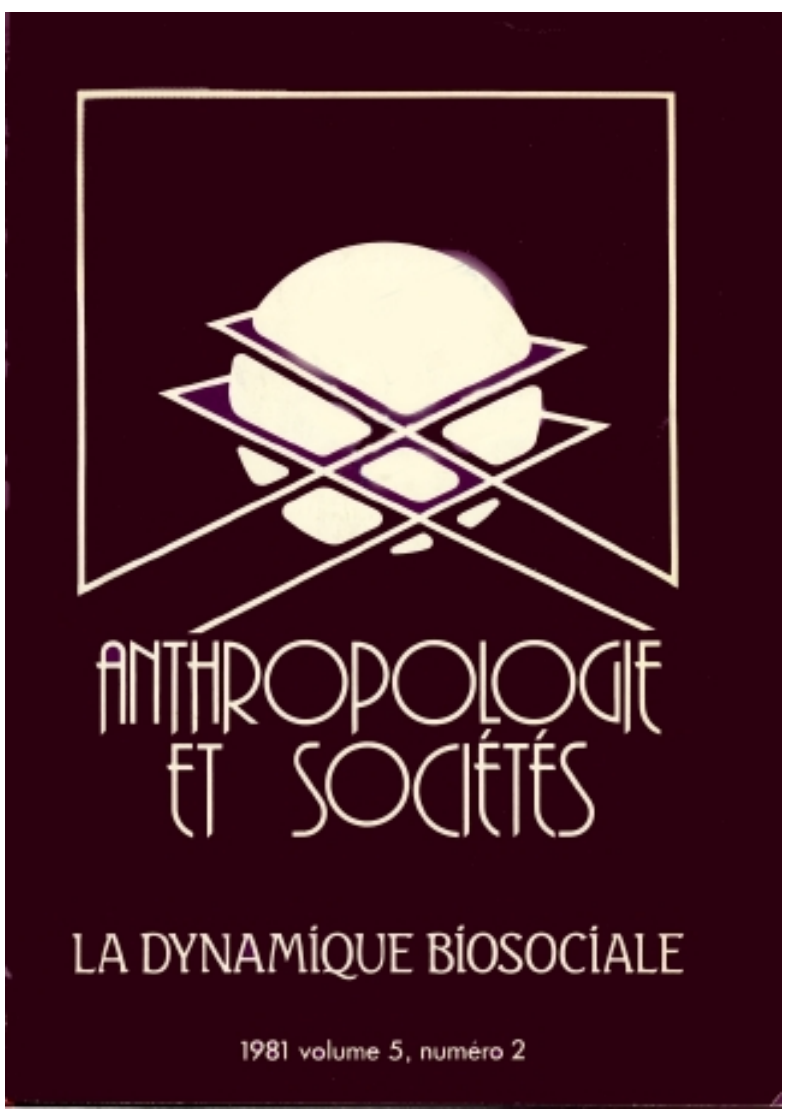

Un article publié dans la revue Anthropologie et Sociétés, vol. 5 no 2, 1981, pp. 155-176. Numéro intitulé : “La dynamique biosociale”. Québec : Département d'anthropologie, Université Laval. 


\section{Table des matières}

Introduction

La tradition des unités

Type

Motif

Trait

Fonction

L'analyse automatique

Actants

Épreuves et modèles de médiation

Eidon

Résumé de la première partie

Les unités de la tradition

Macro-unités

Paragraphes

Micro-unités

Conclusion

Bibliographie 
Elli Köngäs Maranda (1932-1982)

Centre interuniversitaire d'Études sur les Lettres, les Arts et les Traditions (CÉLAT), Faculté des Lettres,

Université Laval (1978)

“La tradition des unités et les unités de la tradition”

Un article publié dans la revue Anthropologie et Sociétés, vol. 5 no 2, 1981, pp. 155-176. Numéro intitulé : “La dynamique biosociale”. Québec : Département d'anthropologie, Université Laval.

\section{Introduction}

L'objet de cet article est d'examiner le développement des recherches sur les caractéristiques des récits folkloriques. On s'intéressera principalement aux travaux poursuivis en Europe et en Amérique du Nord au cours du siècle dernier.

Les considérations suivantes tiendront lieu de point de départ: d'une part, cette donnée qu'on appelle récit n'est pas amorphe. On y trouve au contraire soit des traits à vaste distribution, soit même des traits universels. D'autre part, les récits appartiennent tout aussi bien au domaine linguistique qu'au domaine folkloristique. Ensuite, en tant que données structurées, les récits sont sujets à la classification, on peut en faire la typologie, et on peut les analyser de différentes manières. Enfin, dès que l'analyse se veut comparative, on doit définir les unités d'analyse. Plusieurs de celles-ci, proposées au cours des dernières six ou sept décennies, vont retenir notre attention dans les pages qui suivent. 
On va donc s'attacher à comparer les unités proposées par différents chercheurs. Il ne s'agit pas, cependant, de mettre en question le mérite de ces derniers. Des folkloristes et des anthropologues illustres se sont attaqués au problème des unités. Que les objectifs qu'ils poursuivaient aient été ou non la définition d'unités narratives, et qu'ils aient ou non réussi dans leurs démarches tient en grande partie aux genres de données qu'ils ont analysés. De façon plus précise, ils ont été influencés d'une part par la possibilité qu'ils avaient ou qui leur était refusée d'observer des conteurs en action et par l'urgence des tâches qui les confrontaient, et, de l'autre, par les théories ambiantes ou par celles que, étant donné leur position dans l'histoire des idées, ils étaient en mesure de créer.

Ma proposition est la suivante. Les folkloristes, en tant que tels, ont eu à leur disposition depuis longtemps des textes précieux, soit sous forme imprimée soit sous forme manuscrite dans des archives. Dans bien des cas, une carence de données d'observation faisait la contrepartie de cette richesse de matériaux. Par exemple, Kaarle Krohn commença son travail de collecte a l'âge de 18 ans et commença à publier ses données de terrain cinq ans plus tard: pourquoi ne prêta-t-il aucune attention au contexte vivant, aux idiosyncrasies des conteurs, et à leurs propres théories ? Peut-être, avant tout, parce que la théorie majeure, à l'époque, oblitérait l'importance du conteur individuel pour n'accorder d'intérêt qu'à la conservation des anciennes traditions comme telles. En second lieu, cette même théorie mettait en valeur non pas les procès culturels mais leurs cristallisations dans des produits fixes. Cette position a d'ailleurs été maintenue presque exclusivement pendant plusieurs décennies. En 1946, Stith Thompson écrivait encore : " in contrast to the modern story writer's striving after originality in plot and treatment, the teller of the folktale is proud of his ability to hand on that which he has received "(Thompson 1951 : 4). Et ceci malgré une autre proposition qu'il formule plus loin dans le même ouvrage : "Each version of an oral folktale is different from another » (Ibid. : 368). 
Il y a cent ans que J.G. von Hahn a proposé une formule du conte héroïque, la formule aryenne du départ et du retour (Arische Aussetzungsund Rückkehr-Formel). Cette formule est un mélange des attributs caractéristiques des parents du héros (sa mère est une princesse ; son père est un dieu ou un étranger) ou du héros (l'arrogance du jeune homme), et des actes narratifs (retour triomphant). Otto Rank arrivait à une formule parallèle ; Archer Taylor, dans-son important essai « The Biographical Pattern in Traditional Narrative » (1964) a retracé les étapes de ce développement jusqu'à Propp.

Vladimir Propp (1958) fut le premier à résoudre le dilemme de la stabilité et de la variation, en percevant que tous les contes appartenaient au même type. Milman Parry et Albert Lord (1960) adoptèrent une position semblable, en dernière analyse, lorsqu'ils proclamèrent que toute performance est création. Les deux positions semblent se confondre en ce qu'elles éliminent un des deux termes du dilemme.

On peut ajouter ici une remarque suggérée par les notes de terrain de M.A. Castrén. Grâce à une bourse de la Société Finnoise des Lettres qui encourageait déjà cette collecte, il avait recueilli des contes finlandais dans les provinces de Savo et Carélie en 1839, espérant pouvoir y retrouver la persistance de traits mythologiques. Il se montra cependant déçu parce que les récits strictement « finnois » lui semblaient " être tous des variantes d'un seul thème » (Niemi 1904 : 10). C'était donc en creux, pour ainsi dire, ce que Propp proposerait plus tard.

Il y a bien des rai Sons pour qu'on s'attaque au problème des unités narratives. Une des premières fut le besoin d'ordonner et de classer les récits, surtout à fins documentaires. Une autre vient des frustrations engendrées par les premières taxinomies : celle d'Aarne, par exemple, qui déforme la véritable nature des récits, selon Propp (1968: 11). Une troisième est commune aux soucis des créateurs de ce qu'on désigne comme la "nouvelle ethnographie », surtout celle qu'on identifie avec l'« anthropologie cognitive » ici, le centre d'intérêt est la décou- 
verte des mécanismes tels qu'aperçus de l'intérieur d'un système culturel. Jusqu'à un certain point, les tentatives de Malinowski (1926) pour décrire les catégories génériques des Trobriandais s'apparentent à l'anthropologie cognitive.

Je n'ai pas l'intention de recenser ici un siècle de recherches méthodologiques et théoriques. Mon point de vue est qu'on peut distinguer deux principales classes d'unités, que j'appellerai macro-unités d'une part et micro-unités de l'autre. Les macro-unités ont été proposées par ceux qui ont travaillé sur les problèmes classificatoires à l'intérieur d'une tradition ; quant aux micro-unités, elles viennent de ceux qui essayent de fournir des outils d'application plus générale. J'irais même jusqu'à dire que les macro-unités proposées - comme « type » ne s'appliquent qu'à des récits au sein d'une même famille. D'autre part, quelques-unes au moins des microunités, par exemple le motif, sont mal définies et rendent mal. Enfin, et surtout au cours de la dernière décennie, on a vu éclore plusieurs tentatives qui sont prometteuses de progrès décisif.

\title{
La tradition des unités
}

\author{
Type
}

\section{$\underline{\text { Retour à la table des matières }}$}

Pour comprendre la genèse du concept de type, il faut se reporter au temps où, en Finlande, Antti Aarne poursuivait ses travaux. C'était le tournant du siècle. Depuis 1815, on avait fait d'importantes collectes de contes. La Société Finnoise des Lettres avait été fondée en 1831 à la fin précise de promouvoir la collecte, la classification et l'analyse de la littérature orale. Or les archives de cette société devinrent rapi- 
dement volumineuses ${ }^{1}$. Julius Krohn et son fils Kaarle avaient bien perçu l'importance d'organiser ces archives. Un système approprié devait être créé pour que les archives deviennent une ressource utilisable. C'était là, évidemment, ce qu'on appellerait aujourd'hui un problème de documentation (information retrieval).

Les précurseurs des Catalogues de Types furent des listes de types de contes (Rausmaa éd. 1972) ${ }^{2}$. Kaarle Krohn fit paraître les premières listes finlandaises en 1886. Toutes rudimentaires qu'elles fussent, ces listes n'étaient en aucune façon mauvaises et constituèrent un pas nécessaire vers la définition du catalogue des types qui suivit et qui orienta la recherche sur un plan international par son utilisation de façon inter-culturelle.

Prenons un exemple de la classification de Krohn (1886 : V, 7885) :

61. Le renard comme juge.

62. Le loup comme juge, le renard comme échevin.

63. Que celui qui vient à notre rencontre soit juge.

64. Les trois cours de justice.

65. Les trois témoins.

66. Le renard s'engageant à pleurer sur une Morte.

Une analyse encore plus intéressante est fournie par Krohn (ibid. : XVIII, 412-417) dans le supplément où il donne les variantes que l'on trouvait en 1885 dans les archives de folklore de la Société Finnoise des Lettres. Ainsi, par exemple :

L. Les animaux forestiers ayant peur de la femme.

1 La collection de Kaarle Krohn comprend 18000 numéros (récits et poèmes) ; les collections des archives en 1900 en comprenaient plus de 200000 (Hautala 1968 : 90). Aujourd'hui, les archives contiennent 4 millions de numéros.

2 Les compilateurs avaient connaissance des travaux de Grimm, de Grundvig et de Bolte, selon Taylor (1951). 

a. La leçon de musique de l'ours.
b. Bariolage de l'ours.
c. La castration de l'ours et la recherche de l'onguent.
d. La castration du diable et la recherche de l'onguent.

\section{Respect pour l'homme !}
a. Le vieux lion à son fils.
b. Le boeuf au lion.
c. L'ours au lion.
d. Le lion à l'ours.

Il n'est pas nécessaire de faire l'histoire de la méthode finnoise, dite aussi méthode historico-géographique, qui est bien connue par ailleurs parce que fort adéquatement décrite par Jouko Hautala (1964 : 174349 ; 1968). Laissez-moi seulement dire que les travaux de Krohn et de Aarne s'appuyaient sur une tradition encore vivante; que celle-ci leur fournissait constamment des documents nouveaux; et qu'ils connaissaient eux-mêmes cette tradition vivante dont ils faisaient sur le terrain l'expérience répétée. Il est facile de comprendre le sens- de leurs procédures quand. on songe aux problèmes auxquels ils faisaient face : des données à profusion, qu'il fallait archiver. Ces chercheurs avaient vivement conscience que la responsabilité primordiale était de recueillir et de conserver, pour leurs compatriotes, cet héritage traditionnel. Ainsi sans doute furent-ils étonnés quand, à ce travail qu'ils avaient entrepris pour éclairer une tradition locale, on accorda une importance internationale. La modestie du point de départ et de ses intentions ne laissait pas augurer que plus de vingt catalogues de types utiliseraient la méthode (Thompson 1951 : 413-422). Il en fut un peu de même avec Propp, à vrai dire.

Outre des considérations générales, il faut signaler une des plus importantes caractéristiques du type tel que conçu par Aarne. Ce sont 
des récits dans leur ensemble qu'il analyse. C'est toujours un conte entier qui est son objet.

Disons aussi que le catalogue d'Aarne doit être pris pour ce qu'il est un répertoire, une liste maîtresse pour faciliter l'inventaire. Ceci implique qu'une connaissance préalable, une familiarité est requise, plus importante qu'Aarne ne le croyait. Cette familiarité prérequise est une familiarité avec les données plus qu'avec la taxinomie. Ainsi, en simplifiant : le type $\mathrm{X}$ est ce qui, dans les archives, se trouve sous le numéro indicateur de $X$. Les indications fournies dans le manuel de codage sont peut-être suffisantes pour assigner à peu près correctement de nouvelles données à la classe où elles appartiennent. Mais ceci, seulement si les codeurs connaissent déjà la tradition à cataloguer. Il est significatif à cet égard que les archivistes finlandais aient toujours soutenu que seuls les spécialistes de la littérature orale déjà collectée peuvent classer des données nouvelles. Quand Lauri Simonsuuri occupait le poste de directeur des archives (du milieu jusqu'à la fin des années cinquante), il se faisait bien clair là-dessus : lui, et lui seul, codait tous les documents nouveaux. Cette anecdote montre bien que le système est plutôt pauvre théoriquement ou méthodologiquement, puisqu'il faut vingt ans de travail préalable pour le maîtriser. Et, en dernière analyse, le système, si système il y a, ne saurait être d'aucune valeur générale ou transculturelle.

Le concept de type, ou plutôt les techniques de son usage, ont été notablement améliorés par les Français, l'ouvrage le plus important en cette matière étant le tome Il du Conte Populaire Français où MarieLouise Tenèze a établi le meilleur modèle qui existe d'un catalogue de types (Delarue et Tenèze 1964).

Il faut reconnaître que le conte européen est un phénomène particulier, et que le type est une unité trop grosse pour opérer rentablement par exemple dans l'analyse des récits mélanésiens ${ }^{3}$. Il faut quitter le

3 Des catalogues existent cependant pour l'Indonésie, la Chine et l'Afrique. 
niveau des macro-unités si l'on veut travailler à un niveau transculturel.

\section{Motif}

$\underline{\text { Retour à la table des matières }}$

Stith Thompson, qui a consacré une très longue carrière de chercheur au problème des unités de narration folklorique, a fait face à la question de la validité restreinte d'unités à base européenne. Son premier travail, sa thèse de doctorat à Harvard, en fait, attaquait le problème de la classification des récits nord-amérindiens (Thompson 1929). Cette thèse s'inscrivait dans la tradition de Francis James Child (1892-1898), le classificateur des ballades, et en la compagnie des recherches d'Archer Taylor (1951), autre savant éminent d'outreAtlantique intéressé lui aussi à la classification des documents de littérature orale.

Thompson a traduit le travail d'Aarne en l'augmentant des données contenues dans les catalogues européens rédigés selon le modèle établi par Aarne. L'influence et l'importance du travail de Thompson semblent provenir surtout du fait qu'il a fourni une diffusion plus grande au travail d'Aarne, en traduisant l'original allemand en anglais. Ceci n'est pas pour minimiser la contribution du célèbre folkloriste américain ; on sait bien que, pour obtenir une audience et pour séduire des disciples, il faut publier en anglais. L'intérêt extraordinaire pour Propp après la traduction anglaise de sa Morphologie en témoigne bien.

Donc, Thompson n'a pas créé un nouveau système taxinomique dans son catalogue des types. Il n'en a pas moins éprouvé le besoin d'une révision radicale : "Aarne's general scheme was not disturbed, and all of his typenumbers were retained » (Thompson $1951: 420$ ). 
Même en 1960, en publiant la seconde édition, Thompson écrivait : " With the fewest exceptions, I have made no changes in numbers " (Aarne-Thompson $1961: 8$ ).

Sur la base de son travail sur les traditions indiennes, Thompson a développé un second système, celui des motifs. Il a senti qu'il fallait incorporer d'autres genres que le conte dans le schème classificatoire pour que celui-ci fut valide. Aussi, se lança-t-il dans un projet de grande envergure auquel il consacra trois décennies : faire un catalogue de tous les motifs narratifs, a quelque genre qu'ils appartiennent et de quelque tradition qu'ils proviennent. Le résultat fut publié en 193236, et d'une manière élargie en 1955-58. Il portait le titre plutôt extraordinaire et incroyable de " Motif Index of Folk Literature ; une classification des éléments narratifs dans les contes, ballades, mythes, fables, romans médiévaux, exempla, fabliaux, jest-books, et légendes locales ». Dans l'introduction, Thompson reconnaît que le terme et le concept de motif avaient déjà été utilisé par Albert Wesselski et par Arthur Christensen (1925). Thompson, apparemment, en veut aux intérêts théoriques et, selon ses propres termes, philosophiques, de Christensen. Il s'oppose donc à la définition du motif par Christensen sans en donner d'autre que celle qui tient en une demi-phrase dans son introduction et qui se lit comme suit : « les motifs - ces détails dont sont composés les récits entiers »(Thompson 1932 : 2). D'autres définitions ont été données en passant par Thompson, au cours des années suivantes « motifs (simple incidents having a single point) " (Thompson 1953 : 439) ; "A motif is the smallest element in a tale having a power to persist in tradition » (ibid. : 415). Quoique doive être le motif, on ne nous en dit rien d'autre sinon qu'il est plus petit qu'un récit pleinement constitué. En 1960, Thompson essaie encore une fois de définir le concept de motif en contraste avec celui de type :

Un mot est peut-être souhaitable sur la différence entre un catalogue comme celui-ci (le catalogue des types) et le catalogue des motifs de la littérature orale. Cette classification (des types) s'occupe des récits entiers qui ont une tradition indépendante, ils sont limités à une certaine région. Par ailleurs, le catalogue des motifs est un essai de classification théorique 
des motifs couvrant le monde entier. Parfois (les motifs) sont les équivalents des types, car plusieurs contes sont faits d'un seul motif. Mais l'approche des deux ouvrages est entièrement différente. Ce catalogue-ci n'est qu'une liste pratique des contes pour une certaine région... (AarneThompson 1961 : 8). (Ma traduction).

On dirait que, si le type est « seulement une liste pratique pour une certaine région », malheureusement le motif est seulement une liste impraticable qui ne s'applique à aucune région.

Plusieurs étudiants de Thompson participèrent au travail dans les années cinquante (Baughman, Ikeda, Kirtley), et la seconde édition du catalogue des motifs est, évidemment, encore plus volumineuse que la première.

L'ambition de Thompson était de «réduire à l'ordre le matériau narratif traditionnel du monde entier » (1953 : 93-163), et on peut voir dans son motif la promesse d'une micro-unité d'analyse dont la portée soit assez générale. Mais nous savons que le motif comme outil d'analyse, hélas, est à peu près sans valeur sauf dans les cas où ce que Thompson appelle un motif coïncide en fait avec une autre sorte d'unité. Ainsi, dans les cas des histoires d'Orphée (F81.1) ou de celles de création par plongée (A812) : là, le motif nous est donné comme le bref résumé d'une action dramatique.

À titre d'exemple des problèmes réels posés par la définition du motif par Thompson, je retiendrai le motif A 812, Earth Diver. Thompson le décrit comme suit :

D'un radeau sur la mer originelle, le créateur envoie des animaux pour ramener de la terre (du fond de la mer). Après l'échec de plusieurs, un animal (souvent le rat musqué) réussit. La terre est formée à partir de la parcelle ramenée. (Ma traduction).

Cette description contient, explicitement ou implicitement, les douze motifs suivants : 
A $0 . \quad$ Le créateur.

A $30 . \quad$ Les compagnons du créateur.

A 33. Un animal comme compagnon du créateur.

A 33.2 Un oiseau comme compagnon du créateur.

A 195. Les compagnons de la divinité.

A 196. Les limitations de la divinité.

A 800. La création de la terre.

A 810. L'eau originelle.

A 811. La terre ramenée du fond de l'eau originelle.

A 813. Le radeau sur la mer originelle.

A 814. La terre (faite) de sable jeté sur la mer originelle.

A 830. La création de la terre par le créateur.

Le problème est donc que le motif A 812, par définition un élément minimal, et donc indécomposable, capable d'existence autonome, peut en fait être analysé en éléments plus petits qui, selon Thompson, sont eux aussi capables d'existence autonome. On voit donc que A 812 ne saurait être un élément minimal.

En fait, même les motifs constitutifs en A 812 ont d'autres motifs comme leurs parties. Par exemple :

D'un radeau sur la mer originelle

\section{A 810}

A 813 etc.

Les motifs de cet ordre appartiendraient davantage à la catégorie type, et c'est comme tels qu'ils deviennent utiles pour trouver des données comparatives dans le catalogue des motifs. Le problème majeur posé par le plus grand nombre des motifs du catalogue, c'est que ce sont des débris inutilisables et par conséquent de la pseudoinformation. Si l'on me permet la comparaison, on pourrait caractériser ce que Thompson a essayé de faire au cours de toutes ces années 
par référence à la linguistique : que dirait-on d'un linguiste qui tenterait d'établir la liste de tous les sons bruts (phone types) de toutes les langues du monde, et leurs distributions?

\section{Trait}

$\underline{\text { Retour à la table des matières }}$

L'unité que Thompson utilise dans sa monographie The Star Husband Tale (1953) n'est ni le type ni le motif. Faut-il s'étonner du fait qu'il ne fasse même pas référence au concept de motif quand il entreprend une analyse empirique? Il se sert alors d'une nouvelle unité, qu'il appelle trait, et qu'il définit mieux que le motif. Comparant les intrigues de 86 versions, Thompson trouve 14 points de différence. En conséquence, il utilise ceuxci et les nomme traits. Ce travail appartient évidemment au groupe historico-géographique et il en est de même du concept de trait. De telles définitions ne sont possibles que lorsque l'analyste dispose de plusieurs versions et variantes, et il n'est pas certain que ce concept puisse être validement utilisé lorsqu'il s'agit d'analyser des intrigues autres que celles rencontrées dans un groupe de variantes. En somme, le trait de Thompson est fort différent de son motif, et, soit dit en passant, semble plutôt appartenir à la catégorie des unités " emic » alors que le motif appartiendrait à celle des unités « etic » (Dundes 1963).

Pour bien se rendre compte de la différence entre le trait et le motif, on n'a qu'à comparer le traitement du Star Husband Tale par Thompson dans deux ouvrages différents, Tales of the North American Indians, et sa monographie. L'analyse porte sur l'intrigue dans son entier dans la seconde tandis que l'on n'a que des remarques disparates dans le premier, et elles sont dispersées dans les notes en fin d'ouvrage. 
Il semble donc que, en développant ses motifs, Thompson ait comparé un grand nombre de récits différents où il a essayé de trouver des points de convergence, qui devinrent les motifs ; àl'opposé, quand il eut à écrire une monographie sur les versions d'un récit, il concentra son attention sur les points de divergence, qui devinrent les traits.

\section{Fonction}

$\underline{\text { Retour à la table des matières }}$

Dans l'introduction de sa morphologie, Propp (1958 : 9) critique sévèrement le concept de type élaboré par Aarne. Il s'en prend surtout aux divisions générales faites par Aarne entre contes d'animaux et contes merveilleux.

Je veux faire une remarque initiale : la grande division entre les contes d'animaux et les contes merveilleux ne fut pas annihilée par Propp, son analyse porte seulement sur les contes dits merveilleux, il n'analyse pas de contes d'animaux.

L'argument de Propp repose sur l'importante décision qu'il a prise au départ, à savoir de baser sa classification sur les différences d'actions. Pour Propp, peu importe l'acteur, car seule l'action compte. Ensuite, la fonction proppienne est définie par son impact sur le développement de l'action dramatique ; ainsi, des actes identiques constituent des fonctions différentes selon qu'ils sont causes ou effets. On peut prendre l'exemple chez Propp lui-même, du héros qui reçoit un cheval ou, également, du mariage comme composante de la situation initiale différencié du mariage dans le dénouement.

Je relèverai ici deux problèmes ayant trait aux fonctions proppiennes. Ils ne semblent pas insurmontables. L'un est la dénomination des fonctions. Prenons, par exemple, vilainie et mariage. Vilainie est un substantif qui sert à résumer une relation du type " $x$ lèse $y$ ». Dans la 
plupart des cas y est à la fois le héros du récit et la victime de l'acte de vilainie dont il est question, mais le terme de Propp ne dit rien sur la direction de l'action, c'est-à-dire sur l'orientation de la relation. Pour obtenir cette description plus exacte, il faudrait avoir une représentation des termes, $x$ et $y$, de la direction de la relation et sa nature, ici, vilainie. Et il en va de même pour mariage. Si les fonctions de Propp étaient réécrites de cette façon (cf. Armstrong 1959), elles seraient cadables plus efficacement. Elles en deviendraient sans doute du coup plus nombreuses mais en même temps plus analytiques. Quant à la présentation et au codage des personnages, on peut être plus économe : avant Propp, Axel Olrik avait signalé dès 1909, qu'il est typique de la littérature orale qu'on n'y trouve en général que deux acteurs par scène, une observation que des travaux ultérieurs ont confirmée.

L'autre problème inhérent aux fonctions proppiennes est leur sélectivité. En effet, elles sont loin de décrire la totalité du récit ; en outre, il est difficile de préciser les critères du choix de telle ou telle fonction. Propp lui-même, dans ses analyses, a tendance à reléguer un grand nombre de passages dans la classe fourre-tout des connecteurs quand, encore plus sommairement, il ne les ignore pas tout à fait.

\section{L'analyse automatique}

$\underline{\text { Retour à la table des matières }}$

Je voudrais à présent attirer l'attention sur certains travaux qui, explicitement ou implicitement, directement ou indirectement, peuvent déjà ou pourront éventuellement améliorer la contribution importante de Propp.

L'analyse automatique du folklore, telle qu'elle est pratiquée par Pierre Maranda et son équipe au Canada (Maranda éd. 1974) et par Philippe Richard, Michel de Virville et l'équipe du Centre de Calcul de la Maison des Sciences de l'Homme, par l'équipe du Centre de Cal- 
cul des ATP, et dans quelques autres centres, requiert la formation d'unités analytiques plus stables et rigoureuses que celles dont se satisfont les chercheurs traditionnels. Dans le cadre d'analyses avec le concours d'ordinateurs, il n'est pas nécessaire de charger d'avance les unités analytiques : on n'a pas à prendre de décisions initiales sur celles semblant affecter le plus le cours d'une action. De telles unités peuvent en même temps améliorer le concept des fonctions proppiennes et le concept de mythème proposé par Claude Lévi-Strauss en 1955 (in Sebeok 1968), et leurs groupements tels que révélés par l'analyse automatique correspondraient alors aux "paquets de relations » de Lévi-Strauss.

Il va sans dire que les travaux de Lévi-Strauss continuent de contribuer de façon tout à fait primordiale à une plus grande sophistication de la folkloristique. Comme Pierre Maranda et moi-même l'avons écrit (1971 : 22), certaines oppositions, comme celles du chasseur et de sa proie, du contenant et du contenu, etc., que Lévi-Strauss a bien mises en relief, ont fait progresser nos démarches bien au-delà de ce que pouvaient permettre les motifs de Thompson et les fonctions proppiennes.

\section{Actants}

$\underline{\text { Retour à la table des matières }}$

Alors que Propp prend les fonctions comme point de départ, Claude Bremond propose un système dont le point de départ se trouve dans l'étude des acteurs. Ses unités expriment aussi des relations et sont donc parallèles aux fonctions de Propp. Bremond met l'accent sur l'agent d'une part, sur la victime de l'autre. À chaque point du récit, on peut ainsi imaginer une description de la forme « $x$ pose $a$ par rapport à $y$ ». Ces unités, pour autant que je les ai comprises, ne sont pas prédéterminées, et elles peuvent décrire des récits entiers. 
A.J. Greimas a lui aussi pris en compte l'inventaire des fonctions proppiennes et leur couplage tel que suggéré par Propp lui-même. Greimas constate que « un certain nombre seulement de fonctions se prêtent au couplage ». Il propose ensuite un système plus abstrait : le contrat, l'épreuve, l'absence du héros, l'aliénation, et la réintégration (1966 : 172-221).

L'approche de Greimas est sophistiquée ; on devra se contenter ici de remarquer qu'une telle tentative, à savoir de donner une formulation plus abstraite à des fonctions proppiennes regroupées, n'échappe pas aux types d'objections qu'on adresse à Propp.

\section{Épreuves et modèles de médiation}

$\underline{\text { Retour à la table des matières }}$

L'importance séminale du modèle de Propp ressort clairement du fait que tous les développements récents dans notre domaine sont compatibles avec son approche. Je signalerai ici, chez les Soviétiques (voir Maranda éd. 1974), les travaux de Meletinski et de ses associés. Ceux-ci ont à la fois un champ d'application plus vaste et une organisation structurelle plus précise, que le schème proppien, Au lieu de choisir la fonction comme unité analytique, Meletinski propose celle d'épreuve, les épreuves auxquelles sont Soumis les héros. Il examine la hiérarchie de ces épreuves en fonction des développements séquentiels de J'action. Ainsi, une épreuve préliminaire, $\varepsilon \lambda$ est une étape qui conduit à l'épreuve principale, EL, à une épreuve additionnelle, et à un éventuel rebondissement de l'action.

Meletinski et ses associés ont utilisé cette approche à des fins taxinomiques pour bien distinguer entre mythe et conte européen. À l'Université de la Colombie Britannique, plusieurs chercheurs de notre équipe ont testé le récent modèle soviétique à la fois quant à sa valeur descriptive et quant à son aspect taxinomique. Le modèle a été appli- 
qué à des données Kwakiutl et Okanagan, aux contes de Grimm, aux laïs de Marie de France et aux rituels doukhobor. Les résultats descriptifs s'avèrent supérieurs aux résultats taxinomiques: en d'autres mots, le modèle décrit sur le plan formel mythes et contes mieux qu'il ne les distingue rigoureusement.

Il existe une ressemblance assez remarquable entre les contributions de Meletinski et la concaténation des cinq modèles d'action narrative proposée par Pierre Maranda et moi-même en 1962 (Maranda et Köngäs Maranda 1971 : 36, 2e éd.). Nous avions pris le parti de réduire la hiérarchie des modèles narratifs - types d'action dramatique si l'on veut - à une série de décisions prises par le narrateur. Ce qui peut être économiquement schématisé par une arborescence :

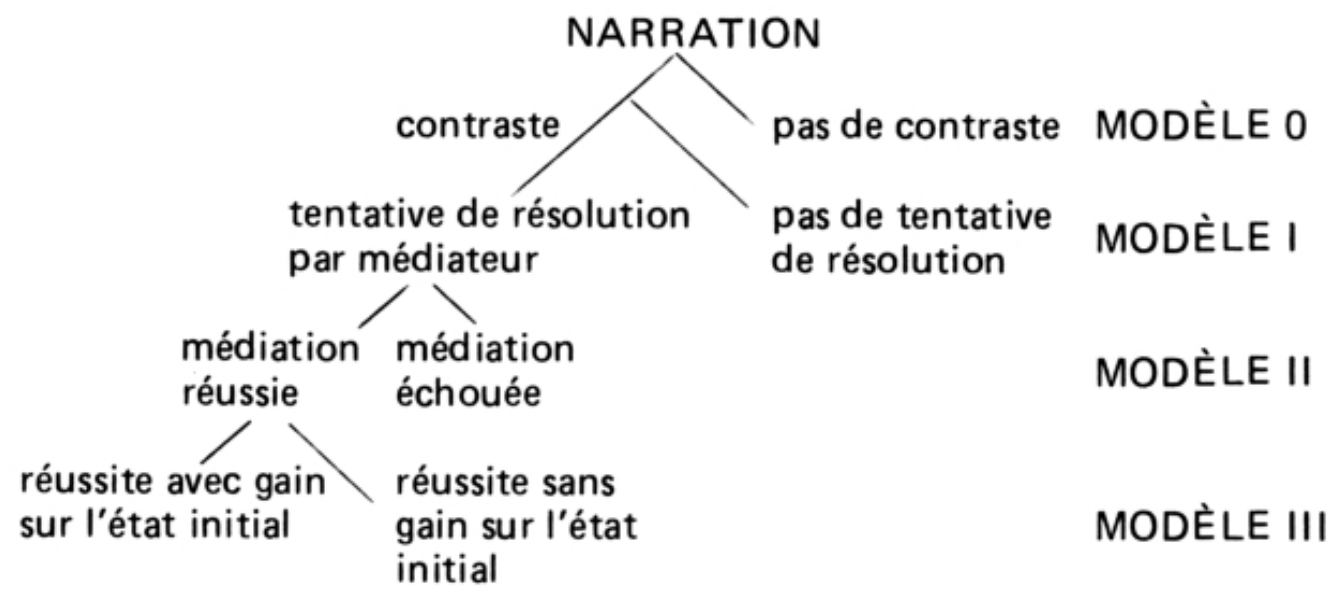

MODĖLE IV

Ainsi. l'épreuve préliminaire de Meletinski se situerait au niveau du modèle II, son épreuve principale à celui du modèle III, et son épreuve additionnelle à celui du modèle IV. En prenant les modèles que nous avons proposés comme des unités constitutives de narration, et en voyant comment celles-ci peuvent s'articuler, il devient clair que le noyau central de l'opération de médiation correspond en fait à l'épreuve principale de Meletinski, avec l'important corollaire suivant : 
la médiation qui échoue correspond à son épreuve préliminaire, et la médiation qui réussit et en outre marque un gain sur l'état initial, correspond à l'épreuve additionnelle et à son résultat. L'isomorphie n'est pas parfaite mais les rapports sont réels.

\section{Eidon}

$\underline{\text { Retour à la table des matières }}$

Encore un autre développement de la fonction proppienne a été proposé par Benjamin Colby (1970a et 1970b). Il a nommé son unité eidon, laquelle, comme l'épreuve de Meletinski, est une unité séquentielle. (Comme telle, elle n'est pas sans ressemblance avec l'épisode, une unité suggérée par quelques chercheurs de l'école historicogéographique, par exemple Anna Brigitta Rooth). L'eidon de Colby comprend les éléments suivants : motivation, engagement, et résolution (MER) et chaque séquence MER est inaltérable - comme on peut s'y attendre, puisque, au moins pour engagement, c'està-dire l'action elle-même, il est nécessaire qu'il prenne place avant la résolution. C'est sur la base de données inuit - récits très largement définis - que Colby a conçu son système et les règles de son développement. Il s'oppose fermement à ce qu'on leur attribue une applicabilité plus générale, mais il est évident que si ses unités peuvent couvrir les récits inuit, elles peuvent aussi couvrir tous les récits dans la mesure où Colby s'attaque aux structures générales de l'action. On notera que Colby considère sa « grammaire du conte inuit » comme une application directe des principes proppiens. 


\section{Résumé de la première partie}

\section{$\underline{\text { Retour à la table des matières }}$}

Bien que toutes les unités examinées jusqu'ici aient été utilisées et donc partiellement testées par au moins un chercheur - et souvent par plusieurs - pour décrire des corpora parfois très vastes, quelques-unes d'entre elles semblent plus productives. Le grand succès du type est bien connu. La raison de ce succès est, selon moi, qu'on l'a utilisé à l'intérieur d'une tradition ou d'une famille (européenne par héritage direct ou par emprunt). Le motif est bien connu, très peu utilisé, et aussi peu utile. Il semble raisonnable de dire que cette première micro-unité s'est avérée inadéquate parce que ce qui est recherché, ce n'est pas le catalogue du contenu d'un conte mais ses éléments minimaux d'information - ces relations minimales - et la façon dont on peut les découvrir. Quant aux unités de taille moyenne, plusieurs sont encore trop récentes, tel l'eidon, pour qu'elles aient été soumises à des tests probants; mais, construites sur une meilleure intelligence des unités, elles semblent augurer un avenir prometteur.

Pour donner un exemple de la comparabilité de certaines des unités présentées jusqu'ici, j'en ferai maintenant une application à une variante française du conte-type 480. Je prends le texte dans Delarue et Tenèze (1964 : 188-189), dont je numérote les phrases (de 1 à 24) pour faciliter les renvois. On peut faire la remarque que, dans le texte tel qu'enregistré, le découpage ne semble pas idéal.

Le découpage que je présente s'inspire de la Morphologie de Propp et d'un travail d'analyse que j'ai fait en 1964 d'une variante anglaise de ce même conte-type (Conte-type 480 dans Aarne-Thompson 1961). 
Retour à la table des matières

ANALYSE

TEXTE PRONONCÉ

1. Situation initiale

1.1. Description initiale

1.1.1. Situation familiale

1.1.2. Description des membres de la famille

(1) Il y avait une femme qui avait deux filles, l'une jolie, l'autre laide.

1.1.2.1.

1.1.2.2.

1.2. Problème initial

1.2.1.

1.2.2.

2. Médiation

2.1. Épreuve primaire

2.1.1. Présentation des acteurs

2.1.1.1. Présentation du preneur d'épreuve

2.1.1.2. Présentation du donneur d'épreuve

2.1.2. Actions

2.1.2.1. Épreuve

2.1.2.2. Succès

2.2. Épreuve secondaire

2.2.1. Épreuve

2.2.2. Succès

3. Résultat

3.1. Récompense

3.1.1. Récompense donnée
(2) La jolie était fort désagréable, désobéissante, boudeuse.

(3) La laide, au contraire était obéissante, aimable, bonne envers tout le monde.

(4) Malgré ses bonnes qualités, sa mère ne l'aimait pas, elle lui faisait faire tout l'ouvrage.

(5) La jolie, au contraire, elle ne faisait rien et sa mère l'aimait beaucoup.
(6) Un jour, la bonne fille fut chercher de l'eau,

(7) elle rencontra la Sainte Vierge qui lui dit :

(8) - Ne voudrais-tu pas me pouiller?

(9) - Très volontiers, répondit la petite.

(10) En la pouillant, la Sainte Vierge lui demanda ce qu'elle trouvait :

(11) - Je trouve des louis d'or, répondit la petite.

(12) - Tiens, lui dit la Sainte Vierge en lui donnant une botte, je te recommande bien d'ouvrir cette boîte en entrant chez 


\subsubsection{Récompense reçue}

4.1. Motivation

4.2. Permission

5. Médiation

5.1. Épreuve primaire

5.1.1. Présentation des acteurs

5.1.1.1. Présentation du preneur d'épreuve

5.1.1.2. Présentation du donneur d'épreuve

5.1.2. Actions

5.1.2.1. Épreuve

5.1.2.2. Échec

5.2. Épreuve secondaire

5.2.1. Épreuve

5.2.2. Échec

6. Résultat

6.1. Punition

6.1.1. Punition donnée

6.1.2. Punition reçue

7. Situation finale vous.

(13) La petite fut fidèle à ce conseil et, en ouvrant la boîte, elle devint jolie, jolie comme le jour, en sorte qu'elle fut plus jolie que sa soeur.

(14) Celle-ci fut jalouse et dit à sa mère qu'elle aussi voulait aller chercher de l'eau.

(15) - Je veux bien, lui dit sa mère, qui était aussi jalouse de la beauté de sa plus grande fille.

(16) La petite fut à l'eau,

(17) elle rencontra, comme sa sœur, la Sainte Vierge,

(18) qui lui demanda aussi si elle voulait la pouiller.

(19) - Tout de même 1 répond la petite d'un air grogneur.

(20) La Sainte Vierge lui demanda aussi ce qu'elle trouvait.

(21) - Je trouve des poux et des puces, répond la petite.

(22) Tiens, lui dit la Sainte Vierge, voilà une botte que tu ouvriras avant d'entrer dans ta maison.

(23) La petite le fit et, en ouvrant la boîte, elle devint laide à faire peur.

(24) La mère en fut affligée et continua de maltraiter sa plus grande et de protéger la plus jeune qui était laide alors. 
Dans son catalogue, Marie-Louise Tenèze présente des analyses monographiques, parallèles à celles de Thompson dans Star Husband Tale, c'est-à-dire des analyses de traits.

Suivant Tenèze on trouve pour cette version :

$$
\begin{aligned}
& \text { I Al (1) IB (2,3,4,5) II Al (6) II C8, Cl3 (7) II C18 (8) III A5 } \\
& \text { (9) - (10) III A6 (11) ?III B8 (12) III B5 (13) - } \\
& (14,15,16,17,18) \text { Il Cl (19) - (20) III C4 (21) III D (22) III } \\
& \text { D5 (23) - (24). }
\end{aligned}
$$

Huit phrases ne sont pas décrites ; quatre phrases sont décrites par une unité, et une phrase a deux descriptions. L'écriture de Tenèze l'emporte de beaucoup sur celle de Thompson dans son catalogue, utilisant des motifs, qui donne :

I. Motif L102. L'héroïne peu prometteuse. L50. La fille cadette victorieuse.

II. H934.3. Les tâches assignées par la belle-mère (mais nous avons ici une vraie mère

III. Rien.

IV. Rien, sinon N825.3. La vieille femme comme aide.

V. Rien.

VI. Rien, sinon Q41.2. Récompense pour avoir purifié une personne détestable (!)

VII. D1860. Rendre beau magiquement (13)

VIII. Q.2. La gentille fille et la méchante $(2,3)$ D1870. La laideur magique (23).

Thompson numérote les éléments (I, II, etc.) et donne les motifs qui sont des variables dans ces catégories. On voit que seulement deux des 24 phrases du conte se laissent décrire avec les motifs dans ce cas. 
Utilisant les symboles et les fonctions de Propp, on obtient, par numéros de phrases :

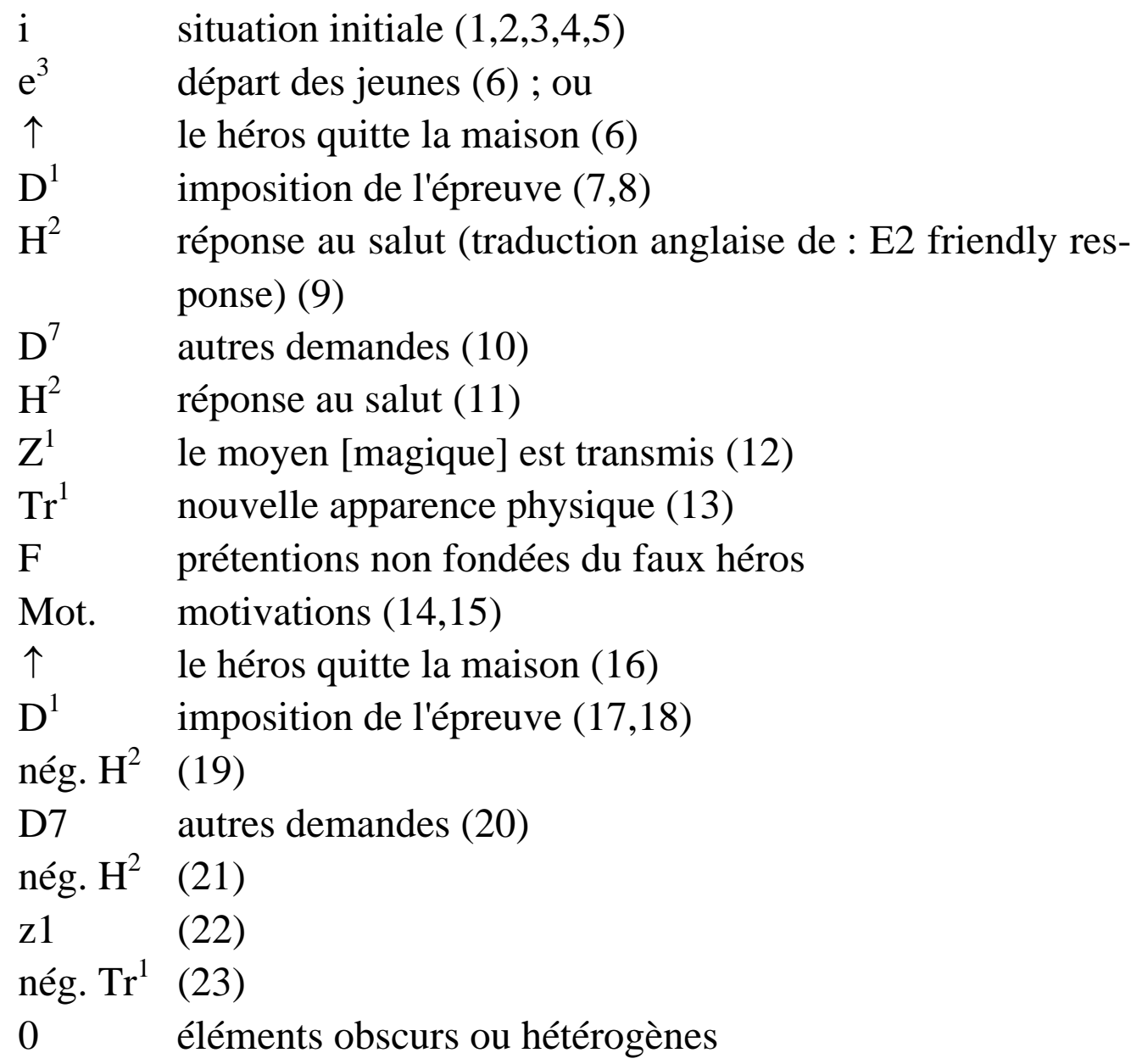

On peut sans doute forcer l'intrigue dans la formule de Propp, mais peutêtre serait-il mieux de désigner (7-11) comme une séquence $\mathrm{M}-\mathrm{N}$ (autrement le conte ne correspond pas à la forme canonique !). M serait alors " difficult task » (et la tâche ne semble pas difficile), et $\mathrm{N}$, " solution (resolution) of a task ». La forme de cette version me semble trop faible pour mériter les descripteurs M-N.

Le problème majeur d'une telle application de Propp est qu'elle peut facilement devenir à contre-sens. Si on fait le découpage et assigne les descripteurs dans un esprit proppien, il faut opter pour la désignation $\mathrm{M}-\mathrm{N}$ ici, parce qu'il existe une relation de causalité dans les 
passages (7-11) et (17-21), laquelle détermine le dénouement. Mais la liste des éléments appropriés pour $\mathrm{M}-\mathrm{N}$ ne contient pas des éléments comparables. En outre, Propp n'a pas très bien élaboré les transformations négatives. Cela se voit bien ici, où nous avons deux contes en miroir et où la seconde héroïne n'est pas frauduleuse mais simplement manquée : elle ne prétend pas duper en passant pour une autre, elle essaye simplement d'émuler l'autre.

\title{
Les unités de la tradition
}

\author{
Macro-unités
}

$\underline{\text { Retour à la table des matières }}$

Toutes les traditions orales connaissent des macro-unités, qu'elles dénomment récits. On ne met pas en doute le point où commence et celui où se termine un récit. Souvent, ces points sont spécialement marqués par des indicateurs formels, telles les formules d'ouverture et de conclusion. En l'absence de ces formules, c'est le style tout entier du récit qui peut le coder de telle façon qu'il soit reconnu par les participants comme fournissant une indication spéciale de ce qui se passe. De même, on trouve que les styles varient avec les genres. Si nous voulions retenir le type pour désigner cette sorte de macro-unités, on pourrait proposer la terminologie suivante (congruente avec l'usage qu'on a fait du mot dans le passé) :

1. Chaque fois qu'une histoire est racontée, on a une nouvelle version ;

2. Chaque fois qu'on observe un changement dans l'histoire, c'està-dire un changement perceptible aux indigènes, on a une nouvelle variante - ici, un indigène dirait que c'est la même histoire, mais racontée différemment ; 
3. Chaque fois que le changement produit dans l'histoire est suffisamment important pour que les conteurs changent le titre de l'histoire ou qu'ils la reconnaissent comme différente d'une autre, on a un type différent. Cependant, l'histoire dans son entier est une unité seulement dans le sens qu'elle a des frontières définies, ce qui, en soi, ne fournit pas d'analyse.

4. Chaque fois que les ressemblances entre des histoires différentes sont suffisamment grandes pour que les indigènes aient un nom pour ce groupe, appelons ce groupe genre - nous rappelant bien qu'il n'y a pas nécessairement de coïncidence entre les genres de cultures différentes. Note : selon ce critère, Aarne avait raison de distinguer les contes d'animaux et les contes merveilleux, puisque la tradition finnoise pratiquait cette distinction ; à cet égard, on peut répondre à la critique adressée par Propp à Aarne qu'il peut être possible d'avoir des structures semblables ou même identiques dans différents genres au sein d'une seule culture. Il en est ainsi, par exemple, dans les matériaux trobriandais de Malinowski, si on les analyse structurellement ou selon ses systèmes symboliques.

\section{Paragraphes}

On a trouvé (par exemple, Dell Hymes, moi-même) que les conteurs utilisent des marqueurs formels d'unités quoique ces marqueurs soient souvent subtils. Un de ces moyens est la répétition de la dernière proposition de ce que j'appelle un paragraphe pour enchaîner, mais sur une nouvelle intonation. A cet égard, Dennis Tedlock (1972) fait maintenant des études raffinées des histoires Zuni.

D'autres façons consistent dans le changement de scène (Olrik 1909) et il ne fait pas de doute que des cultures et des conteurs diffé- 
rents développent une grande variété de ces marqueurs. Dans notre collection mélanésienne, par exemple, ceux-ci varient avec les conteurs. Le point à souligner est que de tels marqueurs 'existent et peuvent être discutés avec les informateurs, les conteurs eux-mêmes et leurs auditoires.

\section{Micro-unités}

J'appelerais ligne la micro-unité typique indigène. Celles-ci sont déterminées par les pauses dans le récit. J'ai marqué ces pauses dans mon travail de collecte à Malaita. En bref, de telles micro-unités forment souvent des phrases simples ou encore elles s'expriment dans la forme d'un verb-phrase ou d'un noun-phrase. Également, le parallélisme, la répétition et d'autres traits stylistiques servent de marqueurs de ces micro-unités.

\section{Conclusion}

\section{$\underline{\text { Retour à la table des matières }}$}

Les chercheurs ont longuement travaillé pour définir des unités d'analyse. On peut tirer une leçon de tous ces efforts, et pas seulement de ceux que je recensais brièvement dans mon exposé. Mais en même temps, la tradition orale elle-même, cette réalité que nous essayons de décrire, d'analyser et de comprendre, au cours de sa vie résurgente au sein de ses manifestations, possède une organisation intrinsèque. Cette réalité que nous étudions est bien réglée et maîtrisée. Le conteur sait conter, l'auditoire sait écouter.

Albert Lord et, avant lui et avec lui, Milman Parry, ont décrit une approche d'unités indigènes comme ils les trouvèrent dans les épopées slaves. Je suis d'accord avec leur perception, sauf qu'elle me semble privilégier des blocs formulaires. Il n'y a pas que les clichés du récit 
qui sont des unités ; les éléments formés plus librement forment aussi des unités indigènes. Les messages qu'on appelle récits sont toujours des messages linguistiques. En conséquence, tous les niveaux linguistiques structuraux s'y trouvent.

Mais le discours folklorique se situe lui-même sur un autre niveau. On pourrait comparer ces deux systèmes de la façon suivante :

\begin{tabular}{cll}
\hline \multicolumn{2}{c}{ Niveaux linguistiques } & \multicolumn{1}{c}{ Niveaux folkloriques } \\
système de règles & \multicolumn{1}{c}{ langage } & tradition \\
performance & discours & narration \\
énoncé & récit \\
macro-unité & phrase & paragraphe \\
micro-unité & morphème & paquets de relations \\
éléments bruts & phone type & relations élémentaires \\
\hline
\end{tabular}

La comparaison n'est pas exhaustive et elle pourrait être développée davantage. Je veux seulement signaler ici qu'il y a une hiérarchie de niveaux semblables, bien que la taille des unités folkloriques soit plus grande.

Qu'en est-il des trésors contenus dans les archives européennes et en général des données de littérature orale qui ont totalement ou presque totalement cessé de vivre dans leur milieu naturel ? Que l'on me permette pour finir de suggérer un projet. Il s'agirait d'étudier des traditions vivantes, là où on les trouve encore, avec les problèmes structuraux à l'esprit. Il faudrait travailler plus lentement et plus soigneusement qu'on a coutume de le faire. Un objectif serait de découvrir le 
système indigène, de développer, comme bien des chercheurs sont en train de le faire, des systèmes théoriques; et de replier l'un sur l'autre ces deux systèmes, pour tester la portée des modèles théoriques. Les archéologues et les anthropologues sociaux travaillent ainsi en collaboration ; les folkloristes et les anthropologues pourraient s'y essayer.

\section{BIBLIOGRAPHIE}

$\underline{\text { Retour à la table des matières }}$

AARNE-THOMPSON

1961 The Types of the Folktale. A Classification and Bibliography. Antti Aarne's Verzeichnis der Märchentypen (FF Communications No 3), traduit et augmenté par Stith Thompson, Foklore Fellows Communications No 184, Helsinki.

ARMSTRONG R.P.

1959 «Content Analysis in Folkloristics », in Ithiel de Sola Pool éd., Trends in Content Analysis. Urbana 1959 : 151170. Publié également dans Pierre Maranda (éd.), Mythology, Penguin Modern Sociology Readings, Harmondsworth, Middlessex 1972 : 173-193.

CHILD F.

1892-98 English and Scottish Popular Ballads, Boston. CHRISTENSEN A. 
1925 Motif et thème. Folklore Fellows Communications No 59, Helsinki.

COLBY B.N.

1970a A Narrative Syntax of Eskimo Folktales, mimeo, Irvine, California.

1970b «The Description of Narrative Structures », in Paul L. Garvin (éd.), Cognition : A Multiple View, New York 1970 : 177-192.

DELARUE P. et $M$. Tenèze

1964 Le Conte populaire français, II, Paris.

DUNDES A.

1963 «From Etic to Emic Units in the Structural Study of Folktales », Journal of American Folklore 75 : 95-105.

1965 The Study of Folklore. New-Jersey : Englewood-Cliffs.

GREIMAS A.J.

1966 Sémantique structurale, Paris.

HAUTALA J.

1964 Suomalainen kansanrunoudentutkimus, Helsinki.

1968 Finnish Folklore Research (1828-1918), Helsinki.

KÖNGÄS MARANDA E. 
1972 Computer Analysis in Folkloristics, ms.

KROHN K.

1886 Suomalaisia kansansatuja /. Eläinsatuja, Helsinki.

LÉVI-STRAUSS C.

1955 « The Structural Study of Myth », in Thomas A. Sebeok (éd,), Myth, A Symposium, Bloomington, 1968 (1re édition: Bibliographical and Special Series of American Folklore Society, tome 5, 1955).

LORD A.B.

$1960 \quad$ The Singer of Tales, Cambridge.

MALINOWSKI B.

1955 « Myth in Primitive Psychology », in Magic, Science and Religion, Garden City (1re édition, 1926).

MARANDA P. (éd.)

1974 Soviet Structural Folkloristics : Text by Meletinsky, Nekludov, Novik and Segal with Tests of the Approach by Jilek and Jilek Aall, Reid and Layton. The Hague et Paris : Mouton.

MARANDA P. et E. Köngäs Maranda

1971 « Structural Models in Folklore », in Köngäs Maranda et Pierre Maranda (éds), Structural Models in Folklore and Transformational Essays. The Hague et Paris : Mouton. 
NIEMI A.R.

1904 Runonkerääjäimme matkakertomuksia, Helsinki.

OLRIK A.

1909 "Epische Gesetze der Volksdichtung », Zeitschrift für Deutsches Altertum, 51. Résumé dans Thompson 1951 : 455-457. Une traduction anglaise a été publiée dans Alan Dundes (éd.), 1965 : 129-141. Une traduction française en a été faite par Ronald Labelle (ms).

PROPP V.

1958 Morphology of the Folktale, Bloomington.

1968 Morphology of the Folktale, 2e édition, Austin.

RAUSMAA P.L. (éd.)

1972 Suomalaiset kansansadut /, Ihmesadut, Helsinki.

ROBERTS L.W.

1959 Up Cuthsin and Down Greasy. Folkways of a Kentucky Mountain Family, Louiseville.

ROOTH A.B.

1951 The Cinderella Cycle, Lund.

TAYLOR A.

1951 English Riddles from Oral Tradition, Berkeley et Los Angeles. 
1964 "The Biographical Pattern in Traditional Narrative ", Journal of the Folklore Institute I : 1/2 : 114-129.

\section{TEDLOCK D.}

$1972 \quad$ Finding in the Center, New ork.

\section{THOMPSON S.}

1932-36 Motif-Index, Helsinki.

1951 The Folktale, New York.

1953 «The Star Husband Tale », Studia Septentrionalia 4 : 93163. Republié dans Dundes (éd.), 1965 : 414-474.

1955-58 Motif-Index of Folk Literature : A Classification of Narrative Elements in Folktales, Ballads, Myths, Fables, Mediaeval Romances, Exempla, Fabliaux, Jest-Books and Local Legends, vols. 1-6, Bloomington (1re éd., FFC, Helsinki 1932-36).

1968 Tales of the North American Indians, Bloomington (1re édition 1929).

TYLER S. (éd.)

1969 Cognitive Anthropology, New York. 